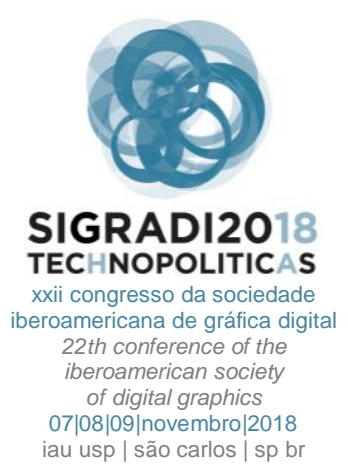

\section{Structural skins over unstable soils}

\author{
Arq. Ana Julia Claro \\ Universidad Nacional del Litoral | Argentina | anajuliaclaro@gmail.com
}

\section{Ing. Enrique Luis Chiappini}

Universidad Nacional del Litoral | Argentina | enriquechiappini@gmail.com

\begin{abstract}
This study aims to research and develop structural support and foundation strategies based on grid systems in city areas with unstable and dispersive soils. It focuses on the possibilities that the structural design with light systems proposed as a technological response/answer to this problem. It takes ravine settlements of Bajada Grande Neighborhood, (Paraná, Entre Rios), as a case-study, pursuing analysis the geotechnical and topographic aspects as initial parameters for the work.
\end{abstract}

Keywords Digital modeling; Structural optimization; Parametrization.

\section{INTRODUCCION}

Los terrenos rivereños acusan una permanente inestabilidad debido a las características topográficas y geotécnicas (deslizamientos, desmoronamientos, baja resistencia del suelo, etc.). Los movimientos en la región del litoral argentino han aumentado su frecuencia durante los últimos 20 años.

Las costas sobre el rio de la ciudad de Paraná (Entre Ríos, Argentina) está conformada por taludes naturales, sobre los cuales, en algunos sectores de la ciudad, se han producido históricamente asentamientos que se ven sistemáticamente afectados por los movimientos que en estos se producen.

Los taludes son superficies inclinadas respecto de la horizontal del terreno, que adopta, de forma permanente, una estructura de tierra. Pueden denominarse laderas cuando se han producido de manera natural.

El estudio de los sistemas estructurales de sostén y de fundaciones, en regiones con estas características, resulta de gran valor debido a la necesidad de dar respuesta a los problemas estructurales e infraestructurales que estos movimientos generan en los asentamientos habitacionales.

Los estudios indican que frente a la presencia de estos tipos de suelos hay dos líneas generales de acción: reducir o eliminar la expansión del suelo y contener sus desplazamientos (costos elevados) o seleccionar un diseño de cimentación apropiado el cual se ve directamente condicionado por el peso que soporta (Patrone \& Prefumo 2002).

En este contexto las estructuras livianas suponen una respuesta viable en las instancias de proyecto en suelos con estas características.

El presente trabajo trata la aplicación de procesos digitales de diseño y análisis estructural de sistemas reticulados de sostén y fundación para dar respuesta a propuestas espaciales sobre suelos inestables. Se propone desarrollar una estrategia de diseño estructural que sea evaluable y responda a las variaciones propuestas por los parámetros característicos del suelo y el programa morfológico, permitiendo integrar el diseño arquitectónico y estructural desde el inicio del proceso, evaluando las respuestas más eficientes.

Los reticulados planos funcionan como una envolvente activa que vincula todos los elementos estructurales de la propuesta y transmite sus cargas hacia nodos, desde donde parten los sistemas de anclajes de fundación propuestos.

El trabajo aborda dos grandes temas. Por un lado, el estudio de los sistemas reticulados planos y los sistemas de fundaciones en suelos inestables (taludes). Por el otro, la inserción del ejercicio proyectual en un sector geográfico determinado, que implica el análisis de las características físicas de dicho sector, cortes geológicos y topográficos del mismo y los aspectos sociológicos del barrio que allí se implanta.

Corresponde aclarar que debido a la especificidad y al amplio desarrollo que cada área temática tiene en sí misma, este artículo propone un recorte que le permita seleccionar los datos pertinentes para abordar las exploraciones y evaluaciones de los procedimientos aplicados en el desarrollo de las estrategias de diseño.

Los avances desarrollados forman parte de estudios realizados para las tesis de maestría y doctorado de los autores, en desarrollo, y los proyectos de investigación "Materialidad Digital en Pieles y Envolventes Arquitectónicas. Ideación y manufactura de prototipos experimentales" y "Diseño Paramétrico de Sistemas Reticulados Espaciales con Optimización Estructural Mediante Algoritmos Geneáticos" dirigidos por el Dr Arq. M. Chiarella (UNL) y el MG. Ing. H. F. Begliardo (UTN), respectivamente. 


\section{DISEÑO ESTRUCTURAL INTEGRADO}

El diseño arquitectónico involucra instancias de determinaciones formales, funcionales y tecnológicas, en un proceso iterativo continuo, hasta arribar a una respuesta superadora de todos los aspectos involucrados. El diseño estructural correcto es el que alcanza la optimización de todas sus variables, cuando se consigue el máximo aprovechamiento de las secciones transversales, la forma geométrica, la distribución topológica y las propiedades del material elegido, y se satisfacen los requerimientos de resistencia, estabilidad y forma de la propuesta arquitectónica inicial. En este proceso entran en consideración múltiples aspectos, dentro de los cuales la eficiencia estructural resulta de vital importancia. Al estar la forma arquitectónica directamente relacionada con la estructura que lo sostiene, el concepto estructural se define cuando la forma queda comienza a establecerse. De esto se desprende que, para controlar el concepto estructural, debemos considerar las consecuencias materiales resultantes al proponer la forma. Para esto resulta necesario aplicar herramientas de diseño y modelado que permitan verificar o modificar su funcionamiento.

Los procesos de diseño que incorporan el criterio estructural desde el inicio se pueden denominar "Diseño Estructural Integrado" ("Structural Integrated Design" SID) (Luyten 2017a). El SID implica que las consecuencias de una decisión arquitectónica aparezcan en el plano de la estructura del diseño y nutran el proceso de diseño arquitectónico con la lógica estructural. El objetivo de este procedimiento no es diseñar una estructura optimizada en si misma o hacer un diseño con una fuerte expresión estructural, o imponer el criterio estructural como condición por sobre los otros criterios. Lo que los diseños estructurales integrados permiten es entender y evaluar el criterio estructural mientras se modela la forma. Lo opuesto es diseñar una forma sin involucrar ningún tipo de conocimiento estructural.

Abordar procedimientos dinámicos y permeables que involucran simultáneamente forma-material-estructura supone un doble desafío. Por un lado, el de superar la perspectiva del diseño digital focalizada sólo en la forma, donde la materialidad no es considerada. Y por el otro, despegarse de la idea que supone a la dimensión material subordinada al diseño. Diseñar una estructura implica la doble acción de evaluar el concepto estructural planteado a través de sus dimensiones. Esta evaluación es cíclica porque lleva a aceptar la estructura o a redefinirla, o incluso rediseñarla. Calcular las dimensiones del concepto estructural es una actividad procedimental que puede ser traducido en una un programa de computadora, donde los input y output se relacionan directamente (Luyten, 2017). La iteratividad se da tanto en el diseño de la arquitectura como de la estructura que la sustenta.

Siguiendo las líneas del SID, y los criterios anteriormente expuestos, poder definir y considerar parámetros que condicionen el funcionamiento de las estructuras en el proceso de definición formal, resulta de vital importancia para este trabajo, que pretende evaluar las respuestas a condiciones de suelo cambiantes. Si se considera que las acciones de definición morfológica y sus consecuencias sobre el conjunto estructural es crítico al inicio del proceso de diseño, desarrollar estrategias verificables que guíen ese camino adquiere gran relevancia.
La definición estructural de la forma desarrolla cada vez mayor potencia de control, definición y modelado. A través de la simulación y evaluación el cálculo numérico computacional de precisión permite nuevos acercamientos integradores de verificación del funcionamiento eficiente de las estructuras a través de las secciones geométricas implicadas.

\section{LA OPTIMIZACION ESTRUCTURAL}

Los conceptos de eficacia y optimización, históricamente vinculados al diseño de maquinarias, aparecen por primera vez aplicados a la construcción, a mediados del siglo XVII (Fontana Cabezas, 2014). En la actualidad se entiende como parte de un diseño estructural correcto la idea de optimización. La cual tiene diversas interpretaciones. Algunos estudios la entienden como la expresión material de las líneas de firmeza, mientras otros plantean un cambio en la interpretación de la materia activa, donde la sección constituye la síntesis de la eficiencia (Gronda \& Chiarella, 2017). El énfasis puede estar puesto en el reticulado o en la sección de los componentes estructurales.

La intención de controlar los costos de la producción motorizó la búsqueda de una organización racional de la materia en las construcciones. A fines del siglo XIX se generalizó la idea de que las entidades físicas, naturales o artificiales están formadas por una materia activa y otra pasiva, es decir, una materia encargada de la estabilidad y resistencia y otra materia sin la cual las exigencias estáticas continuarían verificándose (Cardellach, 1970). A partir de estas observaciones, se inició un proceso continuo de vaciamiento de las secciones resistentes de las antiguas estructuras macizas. Surgiendo la tendencia de expresar materialmente, en las construcciones solamente las líneas de firmeza (Línea de presiones). En los sistemas estructurales si la línea estructural coincide con la fibra media, las componentes de la estructura resultan solicitadas únicamente a esfuerzos de compresión o tracción, optimizándola. Por tales motivos, se entiende que es posible materializar los puntos estratégicos del espacio, mecánicamente, desde la situación más ventajosa para resistir los sistemas de fuerzas exteriores, resultando la forma de la estructura más racional y económica. (Fontana Cabezas, 2014).

El hecho que define la eficiencia estructural por la relación entre forma y propiedades estructurales y materiales ha renovado el interés por las estructuras livianas por parte de la comunidad arquitectónica y de ingenieros (Fritzche, 2013). Los reticulados planos, suponen una estrategia constructiva que en su capacidad de aislar esfuerzos optimiza la respuesta y permite reducir las secciones materiales, pudiendo incluso distribuir más eficientemente las acciones que llegan a las fundaciones.

A partir de los criterios específicos mencionados, a los que se suman los relativos al contexto, se fundamenta la elección de estructuras reticuladas livianas para la materialización de los esqueletos estructurales propuestos en los ejercicios proyectuales. 


\section{CARACTERISTICAS DE LOS SUELOS COSTEROS}

Los procesos geológicos que provocan los movimientos de laderas y taludes son muy destructivos y afectan a las poblaciones en forma directa provocando pérdidas de vidas o heridas de seres humanos, o indirectamente, causando graves deterioros en la infraestructura urbana así como en los asentamientos, industrias, viviendas.

Estudios realizados en la Universidad Tecnológica de Paraná confirman que, en la República Argentina, en particular en el área de las barrancas sobre la costa de la provincia de Entre Ríos del río Paraná, los deslizamientos son bastante comunes y afectan en gran medida a las economías regionales cuando suceden en zonas pobladas. Desde la ciudad de La Paz, al norte, hasta el sur de la ciudad de Diamante, existe un sistema de barrancas que acusa permanente inestabilidad con marcada periodicidad (Cassano \& Bolla 2003).

La problemática de la estabilidad de taludes es una especificidad de la Geotecnia, que, como se anticipó, excede a los estudios abordados en este trabajo. Sin embargo se considera pertinente construir conceptos que permitan dimensionar las características de estas regiones para delimitar las posibilidades de abordaje del trabajo.

La estabilidad puede definirse como la seguridad de una masa de tierra contra una falla o movimiento. En el caso de los taludes naturales, la estabilidad va a estar directamente relacionada a la materia que compone el talud, y a un conjunto de factores que relacionados con la formación del talud, de su historia geológica de las condiciones climáticas sucedidas a lo largo de esa historia, y de la influencia del hombre en ese proceso hasta la actualidad, el flujo de aguas subterráneas, la configuración de los suelos y las rocas del sector, entre otros. (Matteis \& Angelone, 2003).

Cuando se habla de deslizamientos se hace referencia a roturas y movimientos que se producen en el suelo situado por debajo de la masa del talud, los cuales pueden producirse de forma rápida o lenta y con o sin provocación aparente. Pueden producirse por excavaciones al pie del talud, como los provocados por el viento y el rio, entre otros factores. Las fallas más comunes son: los deslizamientos superficiales (creep), los movimientos del cuerpo del talud (falla rotacional y falla traslacional) y los flujos. La estabilidad general del talud requiere de acciones de envergadura, que implica costos elevados y de una especificidad técnica profunda. Entendiendo este marco, se intenta un acercamiento a la problemática de las fundaciones en estos terrenos para dar respuesta a una estabilidad local del suelo comprometido en estos sectores. Cabe mencionar que la vegetación arbórea como la presencia de césped son factores naturales que ayudan a evitar los deslizamientos superficiales, erosión superficial, causada por lluvias y viento (que en algunos casos llegan a ser importantes). Sin embargo, en el sector estudiado, los planos de deslizamiento, así como el círculo de falla de los taludes, se producen a una profundidad mayor, por lo que la presencia de vegetación no afecta ni aporta a mejorar el comportamiento de estos terrenos, al igual que las fundaciones no llegan a dar respuesta a estos movimientos. Por lo que, se aclara que este trabajo busca con el diseño de fundación lograr una estabilización local que garantice la estabilidad de la estructura superior, aunque no del talud.

Los factores que influyen en el comportamiento del talud en cuanto a la estabilidad son:

1. Geometría del talud.

2. Parámetros geotécnicos.

3. Presencia de grietas de tensión.

4. Sobrecargas y cargas dinámicas.

5. Agua freática y flujos.

6. Propiedades tensión - deformación de los estratos presentes

Para este estudio se tomaron en consideración principalmente 2 y 4 . Dentro de los parámetros geotécnicos, se seleccionarán para el estudio de fundaciones con micropilotes el coeficiente de balasto o de compresibilidad y el coeficiente de fricción lateral. Las cargas que actuaran sobre estos sistemas se desprenden de los prototipos modelados y de una sobrecarga de uso determinada para una vivienda.

\section{BAJADA GRANDE}

El Barrio de Bajada Grande presenta un gran número de asentamientos construidos directamente sobre las barrancas costeras del Paraná. Los colapsos y desmoronamientos que sistemáticamente acontecen en estas construcciones determinaron este sector como área de emplazamiento para las experiencias que forman parte de este estudio (fig 1).

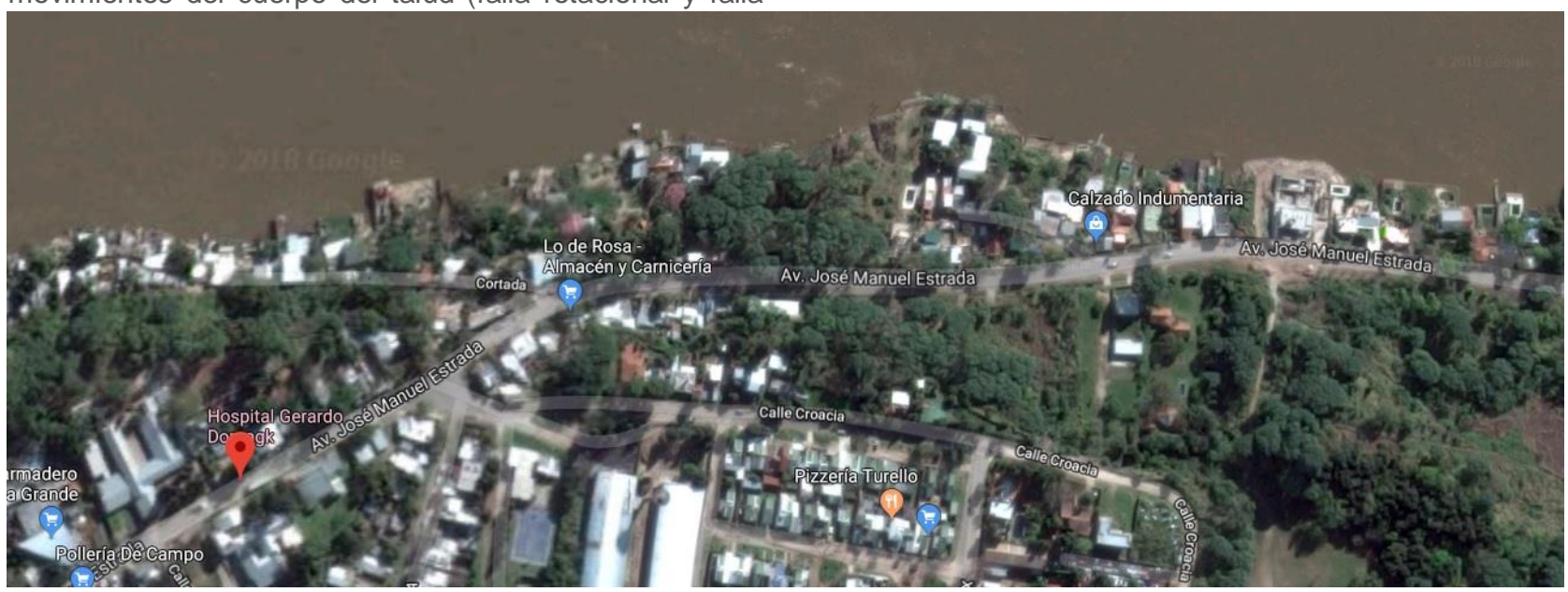

Figura 1: Sector de análisis abordado. Imagen aérea del sector donde se visualiza una de las cortadas y la densidad de asentamientos sobre la costa. Fonte: autores. 
Ubicado en la margen izquierda del río Paraná, limita al noroeste con el río Paraná; al sur, con terrenos anegadizos y al este con el predio de la ex fábrica de Cemento. Se accede a través de dos arterias principales: Avenida Larramendi y Avenida Estrada. Es donde se estableció el primer poblado del territorio entrerriano y de la ciudad. Desde mediados de siglo XIX a la actualidad, en estos terrenos funcionó, un puerto, un servicio de trenes para el transporte de carga, y un servicio de Balsa de Transporte Pesado que unía Paraná y Santa Fe, la fábrica Compañía Argentina de Cemento Portland (1938) y una estafeta de correos (todos ellos actualmente en desuso). Continúan en la actualidad en funcionamiento: la Iglesia Inmaculado Corazón de María (1933), el hospital zonal, una escuela primaria, una escuela secundaria y un centro de educación de adultos con talleres de capacitación laboral. Actualmente la población total estimada es de unos 5000 habitantes. A nivel ocupacional el barrio tiene una población mayoritariamente vulnerable (obreros, de empleados públicos, amas de casa, empleadas domésticas y desocupados). Al ser un barrio costero, tiene también un número importante de pescadores. El barrio sufre varias problemáticas sociales identificadas por sus habitantes, como delincuencia juvenil, falta de seguridad pública y desorganización comunitaria debidos en parte al rápido crecimiento demográfico de la zona, la presencia de asentamientos marginales, la baja calidad y la deficiente actualización de los servicios, en particular los educativos (Mingo 2004).

Sin embargo, y sobre todo en las cortadas ubicadas de cara al rio, puede observarse asentamientos de gran envergadura, viviendas de primer nivel que buscan volcar todas sus visuales al rio. En esta mixtura de situaciones se desarrolla la urbanización de este sector de la ciudad.

Por lo expuesto, y sumado a las características geotécnicas del sector, con inestabilidad del talud sobre la cual se asienta el barrio, que generan el colapso total o parcial de las viviendas particulares y de los circuitos vehiculares y de la infraestructura subyacente, el contexto del barrio se define complejo y delicado.

\section{METODOLOGÍA}

El trabajo presenta los avances alcanzados en las dos primeras etapas de la investigación, donde se abordó la preparación de las situaciones proyectuales base para la generación de modelos de estudio.

Este proceso implicó, en primera instancia, el relevamiento general que permite definir el contexto urbano y social del sector elegido en tres planos: 1. el reconocimiento del sector en sus aspectos materiales: las características de la barranca a través de los estudios de suelo y de corte geológico. 2. las características de las construcciones e infraestructuras existentes, activas y colapsadas (No incluidas en este artículo). 3. las características del grupo social que allí habita en sus aspectos y problemáticas socio- económico, su formación y las acciones y respuestas gubernamentales.

En segunda instancia, se abordó la definición de los parámetros característicos y condicionantes para el trabajo y la delimitación de las herramientas (programas de diseño, análisis y modelado digital), que se presentan a continuación.

\section{PROTOTIPO EXPERIMENTAL}

La propuesta parte de un ejercicio proyectual-morfológico para espacios de pequeña escala que permite explorar la eficacia de los diferentes programas para el diseño y análisis digital de reticulados espaciales, así como los sistemas de fundación, incorporando como uno de los parámetros de condición las características geotécnicas del suelo.

El prototipo de base parte de una estructura reticulada espacial paramétrica de simple capa, que contiene la función del hábitat con cerramientos, cubiertas y divisorios en construcción liviana (Steel framing) y a su vez permite la manipulación espacial debido a que su flexibilidad posibilita la búsqueda morfológica sin dejar de respetar sus condicionantes estático-resistentes.

Para este proyecto se distinguen 2 sectores fundamentales (fig. 2):

- La superestructura (hábitat), liviana, en acero.

- La fundación (raíces), de hormigón armado.

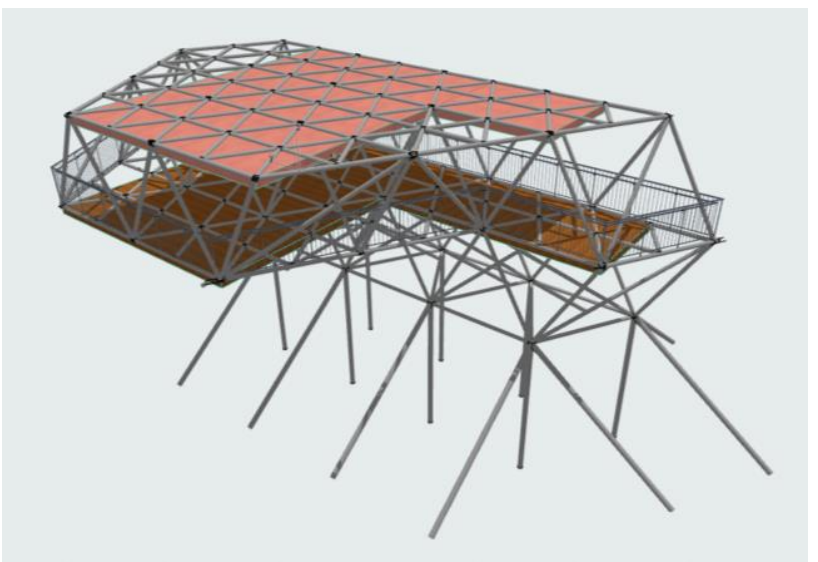

Figura 2: prototipo experimental, superestructura y fundaciones. Fonte: autores.

El primero consta de cubiertas, entrepisos y envolventes, materializadas con reticulados triangulares, planos rígidos en su planos tangentes y resistentes a flexión en los planos normales, y elementos de soporte a tierra, a través de barras verticales e inclinadas de tipo bielas también trianguladas en el espacio. El segundo consta de la fundación o anclaje al talud, materializado por puntos de contacto con el terreno natural a partir de los cuales parten pilotines excavados verticales y oblicuos, cuya resistencia se asigna a su capacidad a compresión o tracción por fricción lateral, o sea por rozamiento entre material del pilote y el terreno (flotantes).

La propuesta se dibuja en CAD elementalmente con líneas y puntos, y luego se graba e importa en un programa de análisis estructural por elementos finitos (FEM), donde se asigna material, secciones, apoyos, etc. Una vez aproximadas las secciones con un dimensionamiento previo, el modelo se exporta con un formato neutro de intercambio BIM, para ser importado en los programas de diseño paramétrico, donde una vez fijado los límites, dimensiones generales y puntos de origen de coordenadas, se procede a la búsqueda de la morfología.

Esta búsqueda es monitoreada en tiempo real ya que el cambio de parámetros implica de inmediato la visualización de resultados de eficiencia estructural, lo 
cual permite decidir siempre con la suficiente información tecnológica estructural específica.

Los programas evaluados y utilizados en la propuesta proyectual son para el modelado: AUTOCAD (Representación asistida), RAM ELEMENTS (Modelado y Análisis Estructural), STRUCTURAL SYNCHRONIZER (Intercambio de Archivos BIM), VISUAL ARQ (Visualizador de Archivos BIM), RHINOCEROS con sus plug-in Grasshopper, (Modelado Paramétrico), Karamba y Galápagos (Análisis Estructural Paramétrico). (figs. 3 a 6).

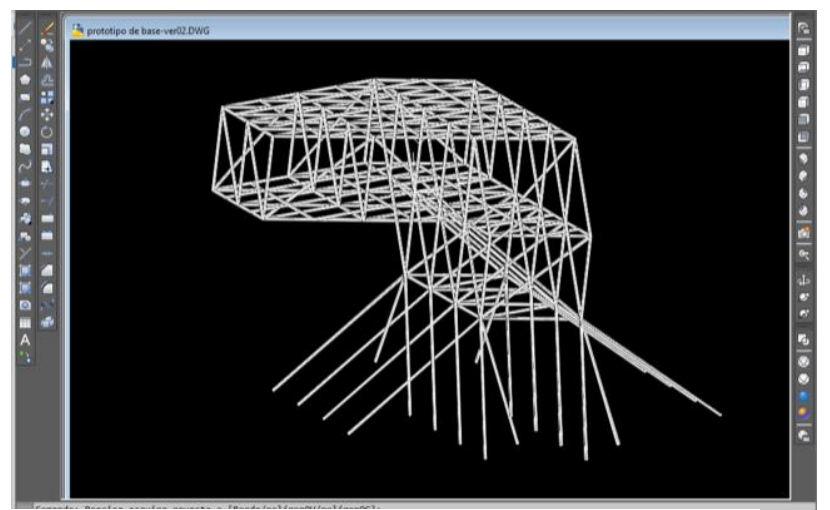

Figura 3: representación asistida (AUTOCAD). Fonte: autores.

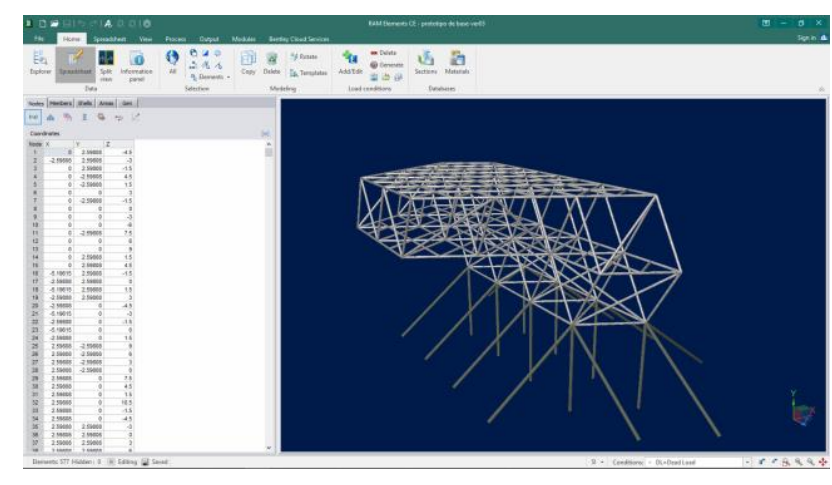

Figura 4: modelado y análisis estructural (RAM ELEMENTS). Fonte: autores.

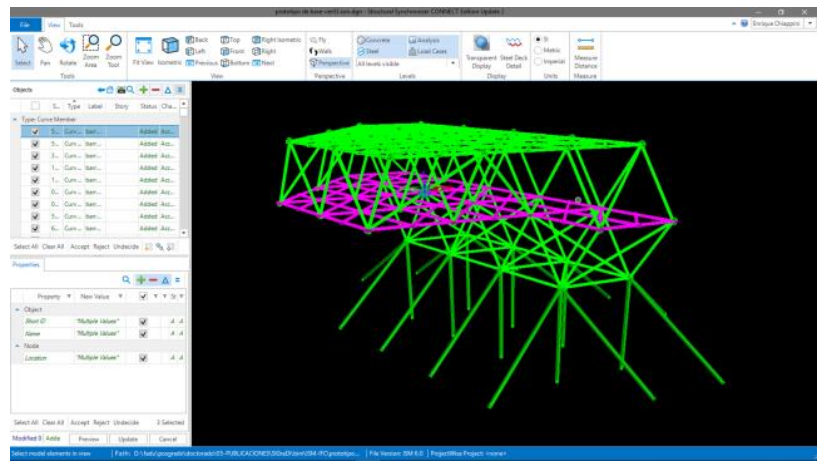

Figura 5: Sincronizador, (STRUCTURAL SYNCHRONIZER). Fonte: autores.

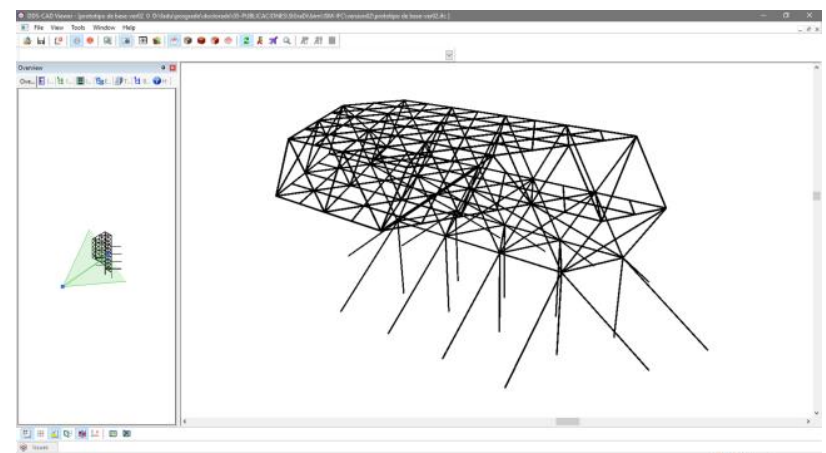

Figura 6: visualizador (VISUAL ARQ). Fonte: autores.

La evolución natural del proceso implica la aplicación de técnicas de la ingeniería estructural en busca de optimización por medio del uso del diseño estructural paramétrico (técnicas de form-finding) y de optimización evolutiva (algoritmos genéticos), (fig.7).

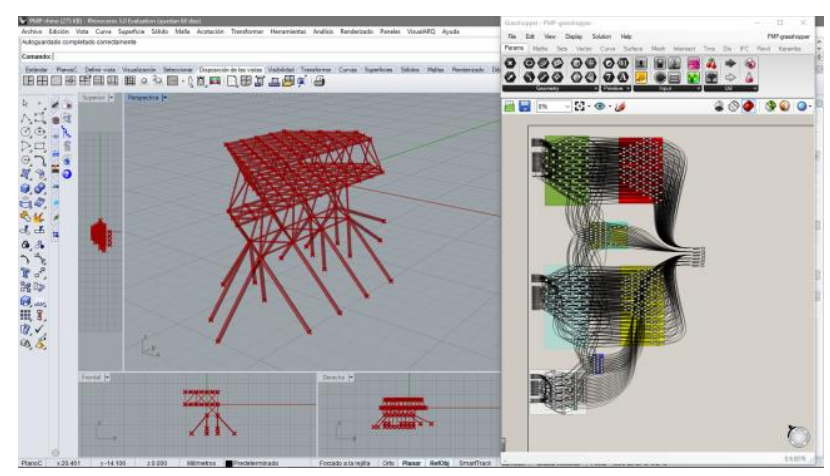

Figura 7: parametrizado (RHINOCEROS, RASSHOPPER). Fonte: autores.

\section{RESULTADOS Y DISCUSION}

Los resultados esperados del presente trabajo de investigación proyectual son el aporte a la construcción de un grupo de herramientas de diseño arquitectónico con fuerte información estructural para considerar su factibilidad tecnológica y su adecuación al contexto de localización. La propuesta se basa en una estrategia de modelado y análisis que integra la forma con la respuesta estructural en un proceso que permite dar resultados en tiempo real. Los prototipos planteados se caracterizan por la dinámica adaptativa de su morfología, la factibilidad tecnológica de su materialización y la sustentabilidad constructiva de su implantación. Si bien el desarrollo específico de modelado es incipiente y se ha realizado un prototipo inicial con baja carga de información de datos como parámetros de condición, la claridad y simplicidad genética de los sistemas estructurales propuestos permiten la evolución del proceso de diseño hasta instancias avanzadas de especificaciones ejecutivas. Debido a las características esenciales de base, el desarrollo de este proceso permitirá definir y ajustar los métodos de fabricación y montaje de elementos. 


\section{AGRADECIMIENTOS}

Agradecemos la colaboración de los Ingenieros Hugo Feliz Begliardo, Matías Bonelli y Arturo Cassano.

\section{REFERENCES}

- Cassano A., Bolla L., Franco J.M., G (2006). "Estabilidad de Barrancas sobre el Rio Paraná. Modelado por métodos numéricos". UTN. Facultad Regional Paraná. Depto de Ing. Civil. ISBN 978-987-25360-6-0. Paraná. Entre Rios.

-Cardellach F. (1970) "Filosofía de las Estructures". Ed. Técnicos y Asociados S.A. ISBN: 9788471460172 . Barcelona.

- Matteis A. Angelone S., 2003 "Estabilidad de Taludes" Geología y Geotecnia. Universidad Nacional de Rosario. Rosario. Santa Fe.

-Fritzche, J.C. (2013). Gridshell Efficiency Opimization. 0748990 Master Thesis. Eindhoven University of Technology. Disponible en:

http:
-Fontana Cabezas J. (2014) "Sobre esqueletos de Gigantes, el paradigma de la complejidad en las estructuras arquitectónicas". Espacio Interdisciplinario. Universidad de la Republica del Uruguay. ISBN: 978-9974-0-1177-9 Montevideo Uruguay

-Patrone J. y Prefumo J. (2002) "La acción de los suelos Expansivos sobre las cimentaciones. Métodos de prevención y control". UM Jornadas de Ing. de Cimentaciones. Uruguay.

-Gronda L. y Chiarella M (2017) "Materialidad Digital. Análisis de estrategias de Arquitectura Orientada al Desempeño transferibles al Diseño Resiliente". SIGraDi 2017 [Proceedings of the 21th Conference of the Iberoamerican Society of Digital Graphics - ISBN: 978-956-227-439-5] 22 24 Noviembre 2017, pp.51-59 Concepción, Chile.

-Luyten L. (2012) "Structurally Informed Architectural Design, Proposals for a Creative Collaboration between Architect and Structural Engineer". Ph. D. Chalmers University of Technology: ISBN 978-91-7385-759-8. Göteborg. Suecia.

-Mingo G. L (2004) "La Pobreza, Condiciones de Vida en la ciudad de Paraná" - Universidad Nacional de Entre Rios. Paraná. Entre Rios. 